\title{
Obesidade e COVID-19: uma revisão da fisiopatologia e exames laboratoriais
}

\author{
Obesity and COVID-19: a review of pathophysiology and laboratory tests
}

Obesidad y COVID-19: una revisión de fisiopatología y pruebas de laboratorio

Weilane Pereira Mota ${ }^{1 *}$, Edislan da Silva Lima1, Mylene Silva de Sousa1, Raissa Freitas Barbosa ${ }^{1}$, Jhonys Ribeiro Mota Berlatto ${ }^{1}$, Esdras Welesson Matias de Sousa ${ }^{1}$, Karen Vitória Lima Silva ${ }^{1}$, Vitória Estefany Carvalho Dutra1', Fernanda Farias Costaㄹ, Antônio Carlos Melo Lima Filho'1.

\section{RESUMO}

Objetivo: Descrever a fisiopatologia da obesidade e evidenciar a importância dos principais exames laboratoriais na avaliação de pacientes obesos com COVID-19. Revisão bibliográfica: A infecção causada pelo SARS-CoV-2 traz consigo um grande espectro de sintomas nos pacientes, variando de assintomáticos, sintomas inespecíficos e/ou até o óbito. Dentre as principais complicações associadas aos óbitos por COVID19 estão a esteatose hepática, diabetes mellitus, cardiopatia e doença renal, que na maioria das vezes, estão ligadas à obesidade. Os testes laboratoriais são realizados através da dosagem de enzimas específicas para cada patologia, como exemplo a aspartato aminotransferase (AST), lactato desidroganease, troponina e creatinina quinase cardíaca para de avaliação de cardiopatia, e hemoglobina glicada e o teste de Tolerância a Glicose para níveis de glicemia. Por sua vez, podem ainda ser avaliados a função hepática por meio da Gama-Glutamil Transferase e lesão do órgão pela AST e a alanina aminotransferase. Dosagens de ureia, creatinina, avaliação da taxa de filtração glomerular e os elementos do sedimento da urina podem ainda ser solicitados para avaliação renal. Considerações finais: Os exames laboratoriais devem ser interpretados com cuidado, bem como o paciente deve ser acompanhado por uma equipe multidisciplinar, a fim de que tenha um melhor prognóstico.

Palavras-Cchave: COVID-19, obesidade, doenças metabólicas, estresse metabólico, SARS-CoV-2.

\begin{abstract}
Objective: To describe the pathophysiology of obesity and highlight the importance of the main laboratory tests in the evaluation of obese patients with COVID-19. Bibliografic review: The infection caused by SARSCoV-2 brings with it a wide spectrum of symptoms in patients, ranging from asymptomatic, nonspecific symptoms and/or even death. Among the main complications associated with deaths from COVID-19 are hepatic steatosis, diabetes mellitus, heart disease and kidney disease, which, in most cases, are linked to obesity. Laboratory tests are performed by measuring specific enzymes for each pathology, such as aspartate aminotransferase (AST), lactate dehydroganease, troponin and cardiac creatinine kinase to assess heart disease, and glycated hemoglobin and the Glucose Tolerance Test for blood glucose levels. In turn, liver function can also be evaluated by means of Gamma-Glutamyl Transferase and organ damage by AST and alanine aminotransferase. Measurements of urea, creatinine, assessment of glomerular filtration rate and urine sediment elements can still be ordered for renal assessment. Final considerations: Laboratory tests must be interpreted with care, and the patient must be monitored by a multidisciplinary team, in order to have a better prognosis.
\end{abstract}

Keywords: COVID-19, Obesity, Metabolic diseases, Metabolic stress, SARS-CoV-2.

1 Universidade CEUMA (UNICEUMA), Imperatriz - MA *E-mail: weii.mota@hotmail.com 


\section{RESUMEN}

Objetivo: Describir la fisiopatología de la obesidad y resaltar la importancia de las principales pruebas de laboratorio en la evaluación de pacientes obesos con COVID-19. Revisión bibliográfica: La infección causada por el SARS-CoV-2 trae consigo un amplio espectro de síntomas en los pacientes, que van desde síntomas asintomáticos, inespecíficos y / o incluso la muerte. Entre las principales complicaciones asociadas con las muertes por COVID-19 se encuentran la esteatosis hepática, la diabetes mellitus, las enfermedades cardíacas y las enfermedades renales, que, en la mayoría de los casos, están relacionadas con la obesidad. Las pruebas de laboratorio se realizan midiendo enzimas específicas para cada patología, como aspartato aminotransferasa (AST), lactato deshidroganasa, troponina y creatinina quinasa cardíaca para evaluar la enfermedad cardíaca, y la hemoglobina glucosilada y la glucosa. Prueba de tolerancia para los niveles de glucosa en sangre. A su vez, la función hepática también puede evaluarse mediante la gamma-glutamil transferasa y el daño orgánico por AST y la alanina aminotransferasa. Aún se pueden solicitar mediciones de urea, creatinina, evaluación de la tasa de filtración glomerular y elementos del sedimento urinario para la evaluación renal. Consideraciones finales: Las pruebas de laboratorio deben interpretarse con cuidado y el paciente debe ser monitoreado por un equipo multidisciplinario, para tener un mejor pronóstico.

Palabras clave: COVID-19, Obesidad, Enfermedades metabólicas, Estrés metabólico, SARS-CoV-2.

\section{INTRODUÇÃO}

Com os primeiros relatos de casos feitos por Zhu, et al. (2020) na província chinesa de Wuhan em dezembro de 2019, a COVID-19 foi estabelecida como uma síndrome respiratória aguda de causa inespecífica. Atualmente, sabe-se que o SARS-CoV-2 é o agente etiológico viral, sendo ele de RNA fita senso positiva, pertencente à família do Coronavírus. Assim como as infecções causadas pelo SARS-CoV e MERSCoV, causadores da síndrome respiratória aguda (SARS) e síndrome respiratória do Médio Oriente (MERS), há complicações respiratórias, entretanto, durante a infecção é também comum um grande espectro de sinais e sintomas, que compreende desde pacientes assintomáticos à sintomas inespecíficos. Dentre os sintomas inespecíficos exemplificam-se a tosse, febre, dor de cabeça, perda do olfato, perda de paladar, fadiga, mialgia, mal-estar geral e diarreia (XU Z, et al., 2020).

De acordo com a piora do quadro, há instauração do estresse oxidativo celular e formação de um processo inflamatório intenso, caracterizado pela liberação de fator de necrose tumoral alfa (TNF- $\alpha$ ), interleucinas 1 (IL1), 6 (IL-6), 8 (IL-8) e interferons, capazes de intesinficar ainda mais a resposta inflamatória contra o vírus em forma de loop. Entretanto, esta resposta exagerada não é benéfica para o corpo do paciente, devido a grande quantidade de citocinas pró-inflamatórias no paciente, resultando no evento que é conhecido como tempestade de citocinas (TAY MZ, et al., 2020; HU B, et al., 2021). Após a entrada do vírus nas células do hospedeiro por meio da ligação com o receptor ACE2, há uma piora nos tecidos extrapulmonares e sobrecarga do sistema imune, levando muitas vezes ao colapso associado a tempestade de citocinas e estresse oxidativo descontrolado (SILVA CC, et al., 2021).

Até março de 2021, foram registrados mais de 130 milhões de casos confirmados de infecção pelo SARSCoV-2 no mundo, sendo mais de 2 milhões de óbitos relacionados à COVID-19. No Brasil, a infecção vem ganhando cada vez mais importância, uma vez que o país ocupa o lugar de segundo maior em número de casos no mundo. Desta forma, atualmente, o país é responsável por quase 13 milhões de infectados e mais de 300 mil mortos e apontam ainda que dentre as principais complicações associadas aos óbitos por COVID19 no país estão à diabetes mellitus, hipertensão, cardiopatia e doença renal, além da obesidade (MINISTÉRIO DA SAÚDE, 2021).

De modo geral, os pacientes obesos têm maior risco de hipertensão, diabetes mellitus, complicações renais e cardíacas, sendo todas estas complicações relacionadas às mortes por COVID-19 (COLARES VS, et al., 2020). Dados apontam ainda que $16,5 \%$ dos brasileiros são dislipidêmicos, ficando com valores próximos aos norte-americanos, que são $12,9 \%$, e abaixo da população chinesa, com $36,5 \%$. Este tal 
resultado aponta que a preocupação acerca da obesidade é bastante palpável e preocupante em todo o mundo, destacando-se ainda o fato de que a mesma predispõe o corpo humano à complicações mais severas causadas em infecções oportunistas, tais como muitas doenças virais (SOUSA ELH, 2020).

Desta forma, ressalta-se mais uma vez a importância do estudo deste grupo de risco por meio de uma equipe multidisciplinar, que empregue tanto a clínica quanto laboratorial em suas abordagens, visando o planejamento de estratégias específicas para cada paciente. Em suma, este artigo teve como objetivo principal descrever a fisiopatologia da obesidade, ressaltando a importância dos principais exames laboratoriais realizados rotineiramente nos laboratórios, visando o melhor manejo do paciente obeso com COVID-19.

\section{REVISÃO BIBLIOGRÁFICA}

\section{Obesidade}

Com o passar das décadas, a obesidade tornou-se um dos maiores problemas de saúde em todo o mundo. De forma geral, ela pode ser entendida como o excesso de gordura corporal, sendo esta uma condição associada à transtornos de alimentação e, principalmente, aos hábitos de vida inadequados. Além disso, destaca-se que ela pode ainda ter caráter genético, por meio da herança de enzimas com menor capacidade metabólica (BRAY MS, et al., 2016; JONAS AO, 2018). Corroborando com esta ideia, sabe-se que além de hábitos alimentares prejudiciais e a falta da prática de exercícios físicos, as estimativas indicam que o risco de herdabilidade de índice de massa corporal elevado varia entre 40 a 70\% (BRAY MS, et al., 2016).

Além disso, a obesidade também chama atenção devido a sua associação com a síndrome metabólica, condição esta em que o paciente apresenta o desequilíbrio no sistema metabólico, sendo uma condição comum entre os idosos e pacientes hospitalizados. Seguindo a tendência mundial, no Brasil, observou-se o aumento de 19,8\% no número de obesos em 2018. Ainda em consonância com o padrão mundial, esses pacientes apresentam risco elevado de desenvolvimento de doenças cardíacas, renais crônicas, acidente vascular cerebral, diabetes tipo 2, esteatose hepática e hipertensão (OLIVEIRA JRB, et al., 2020).

Por estes motivos, a prevenção assim como o tratamento para a obesidade deve-se incluir a reeducação alimentar associada a exercícios físicos diários. Além disso, em casos extremos, pode-se ainda inaçar mão de medidas invasivas como a cirurgia bariátrica ou até mesmo farmacoterapia de intervenção. Outras formas de cuidado como investimento em medidas de melhora socioeconômica da população em geral, visando reduzir o consumo de alimentos ultra-processados e realização de atividades físicas devem ser aplicadas para que o índice de óbitos não tenha crescimento (KIRCH JR, 2018).

Patologicamente, há acúmulo gradual de adipócitos e/ou gotículas lipídicas nos tecidos do indivíduo obeso, levando o aumento do sobrepeso (Figura 1). Órgãos como o fígado e músculos esqueléticos desenvolvem quadro de acúmulo de gotículas lipídicas citoplasmáticas, dando início ao estresse metabólico nestes tecidos (HEYMSFIELD SB, et al., 2014).

De acordo com o esquema, hábitos inadequados, juntamente com fatores genéticos e transtornos alimentares levam ao desenvolvimento da obesidade. Por sua vez, a mesma é caracterizada pelo excesso de gordura corporal, observada no fígado na forma de esteatose hepática, e pela formação de radicais livres por todo o corpo. Além disso, esses radicais formados em excesso levam à instauração do estresse oxidativo, responsável por causar danos às proteínas, quebra do DNA e desestabilização da membrana plasmática. Tais alterações resultam em perda de função e morfologia celular normal, causando asma, infertilidade, perda do sono e cardiopatias (KIRCH JR, 2018).

Outro ponto importante trata-se da dislipidemia, desequilíbrio da pressão arterial, formadoras de doença renal e diabetes tipo 2, ambas comprometedoras da saúde do obeso. Em amarelo fatores importantes para desenvolvimento da obesidade; em azul, alterações intracelulares; em rosa, patologias associadas ao desenvolvimento do quadro de obesidade (HEYMSFIELD SB, et al., 2014). 
Figura 1 - Desenvolvimento das doenças associadas à obesidade.

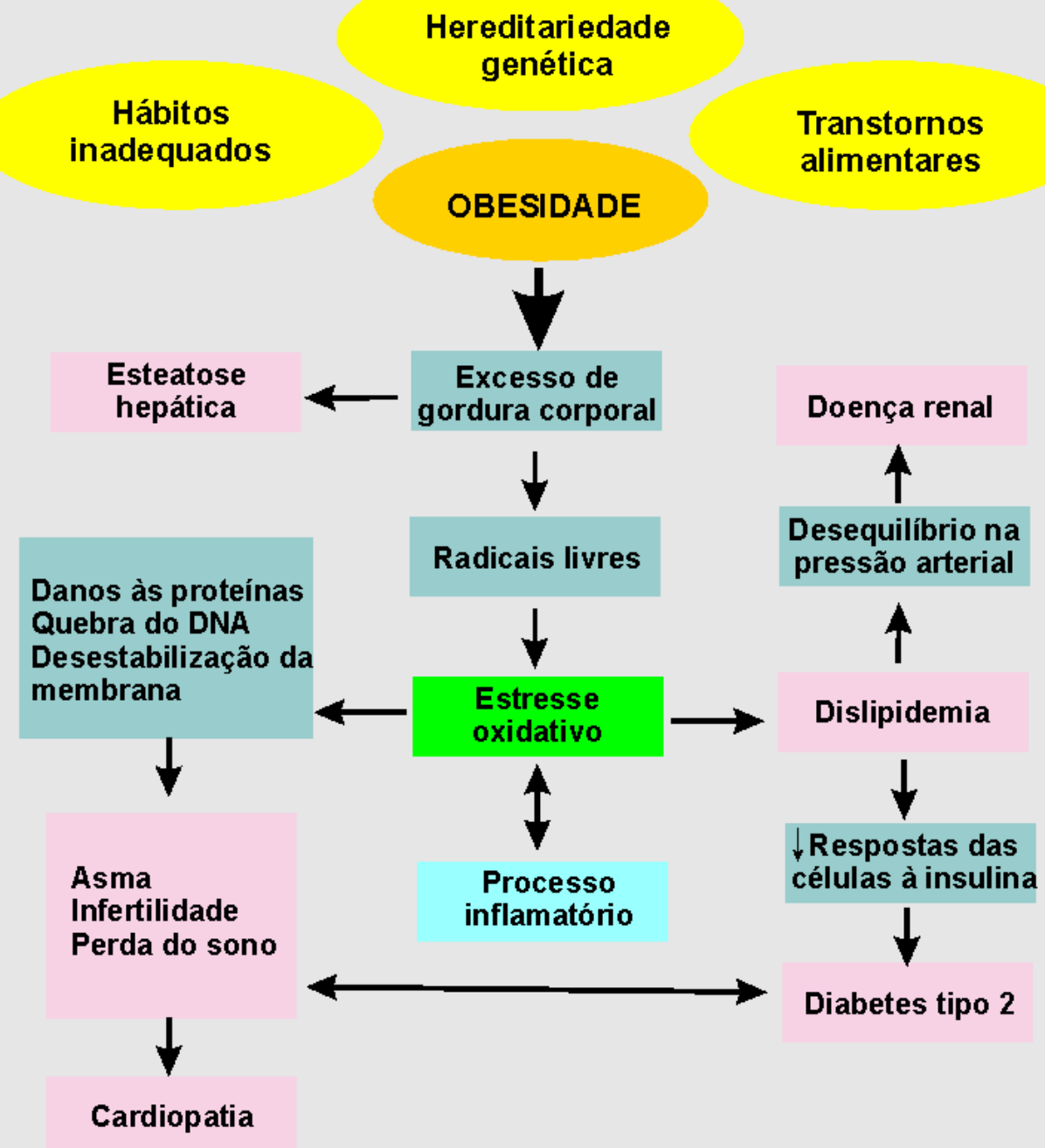

Fonte: Mota WP, et al., 2021, baseado em: Manna P e Jain SK, 2015; Heymsfield SB, et al., 2017; Kirch JR, 2018.

No fígado, este evento dá início a esteatose, resultando no comprometimento da capacidade hepática de síntese e metabolização. Em seguida, como resultado da alteração das atividades normais celulares, há um aumento de radicais livres acima dos níveis regulados pelas enzimas antioxidantes endógenas, e a instauração do processo inflamatório devido o aumento da atividade dos adipócitos e macrófagos dentro do tecido adiposo. Neste mecanismo há ainda o excesso da liberação de citocinas pró-inflamatórias e de ácidos graxos como consequência da hidrólise de triglicerídeos nas células que se direcionam para o plasma (HEYMSFIELD SB, et al., 2017).

É sabido que o processo oxidativo instaurado é responsável ainda por levar a perda da função de diversas proteínas devido à alteração da forma, bem como à danos diretos ao DNA e desestabilização da membrana plasmática. Tal fato pode ser uma das vias responsáveis pela formação de asma, resistência à insulina, infertilidade, perda da qualidade do sono e doenças cardíacas, por exemplo (MANNA P, et al., 2015). Deste modo, associado ao excesso de radicais, os altos níveis de lipídeos circulantes, em um quadro conhecido como dislipidemia, resultam na diminuição da resposta das células à insulina, correspondente aos achados de diabetes tipo 2 (KAUR JA, et al., 2014). 
Assim, em resposta aos eventos lesivos celulares causados pela dislipidemia e desbalanço inflamatório e oxidativo, há comprometimento da vascularização e desequilíbrio da pressão arterial. Em um passo a seguir, a hipertensão instaurada pode comprometer a função renal, causando ainda derrame e doenças cardíacas (HEYMSFIELD SB, et al., 2017). Visto a grande variedade de condições associadas à obesidade, bem como o alto número de obesos no Brasil, torna-se importante estudar esta população de risco, visando o desenvolvimento e aprimoramento de estratégias de prevenção, tratamento e melhor manejo deste grupo.

\section{Importância da atenção aos resultados dos marcadores laboratoriais na avaliação do obeso}

Assim, de acordo com a Sociedade Brasileira de Patologia e Análises clínicas, os exames laboratoriais servem no auxílio da tomada de decisões médicas no tratamento, bem como acompanhamento de pacientes. Atualmente, os exames laboratoriais são utilizados na prática da análise do índice de massa corpórea e avaliação laboratorial do obeso com COVID-19. Além dos usuais testes de PCR e sorológicos para diagnóstico de infecção viral, é imprescindível a avaliação de diversos marcadores laboratoriais para cardiopatias, hepatopatias e diabetes, por exemplo, destacam-se as dosagens de diversas proteínas de lesão e morte celular (FÖLDI M, et al., 2021).

Desta forma, sabe-se que a existência de uma doença cardiovascular é um fator de risco importante durante o COVID-19, uma vez que a proteína $S$ do vírus pode interagir com a enzima conversora de angiotensina 2 no corpo humano (NISHIGA M, et al., 2020). Desta forma, é indicativo que sejam realizados exames laboratoriais de acompanhamento e avaliação do coração do paciente com COVID-19. Assim, a maioria dos testes laboratoriais realizados para a avaliação de cardiopatia são representados pelas dosagens das enzimas aspartato aminotransferase (AST), lactato desidroganease (LDH), troponina e creatinina quinase cardíaca (CK-MB), por exemplo.

De acordo com a Sociedade Brasileira de Cardiologia (SBC) (2014), a AST e LDH são enzimas que participam do metabolismo celular, sendo também encontradas em diferentes tecidos, e por este motivo, não são consideradas como marcadores específicos de lesão cardíaca. Na prática, as mesmas podem ser liberadas depois da prática de exercícios físicos intensos, assim como em lesões hepáticas agudas e crônicas, fato que não demonstra necessariamente uma cardiopatia presente no corpo do paciente (SANTI MC, et al., 2018).

Por sua vez, a dosagem de CK-MB e troponina são os exames mais específicos do coração, devido à alta sensibilidade e especificidade desses marcadores. A CK-MB facilita o diagnóstico precoce quanto o tardio e 0 aumento da troponina pode ser identificado em pequenos infartos mesmo que não haja elevação da CKMB. Um exemplo é a CK-MB está elevada durante o infarto do miocárdio em mais ou menos 6 horas após a lesão (SBC, 2014; SBC, 2015; SUS-BH, 2016). Assim, destaca-se mais uma vez que pacientes obesos tem maiores chances de desenvolvimento de problemas cardíacos, sendo este um fator de complicação na COVID-19. Por este motivo, as dosagens de troponina e CK-MB, por exemplo, devem ser realizadas de forma rotineira nestes pacientes, observando-se a extensão e gravidade da lesão para melhor acompanhamento (CERCATO C e FONSECA FA, 2019).

No mesmo raciocínio, os pacientes obesos em sua maioria apresentam também a Diabetes Mellitus. De acordo com a Sociedade Brasileira de Diabetes (2019), em 2045 pode haver mais de 628,6 milhões de pessoas vivendo com a mesma, o que ressalta a importância das medidas preventivas contra a obesidade, por ser um fator de risco para esta patologia, assim também cuidados redobrados de pacientes que possuem uma predisposição genética. Fisiopatologicamente, os diabéticos possuem resposta imune prejudicada, e da mesma forma, a piora na cicatrização (REIS MAOM, et al., 2021).

Desta forma, segundo a Sociedade Brasileira de Diabetes (2019) torna-se de extrema importância a realização dos exames laboratoriais em pacientes acometidos pela diabetes e simultaneamente pelo COVID19. Dentre os exames solicitados para a detecção da patologia estão a avaliação da glicemia após 8 a 12 horas em jejum e a hemoglobina glicada ( $\mathrm{HbA} 1 \mathrm{c})$, que é uma medida indireta de glicemia e representa uma média dos últimos 4 meses dos níveis glicêmicos do paciente. 
Da mesma forma, outro exame importante que pode ser solicitado é o de Tolerância a Glicose (TOTG). No TOTG o paciente ingere $75 \mathrm{~g}$ de glicose, sendo coletadas amostras consecutivas por 2 horas, buscando avaliar a curva glicêmica e consequente capacidade de metabolização deste carboidrato. Contudo, a relevância da solicitação dos exames citados é extremamente necessária para que se tenha um diagnóstico definitivo e que promova uma conduta de tratamento eficaz e de sucesso, que limita a progressão da doença e possibilite a alta para o paciente (SBD, 2019; SUS-BH, 2016).

Outro órgão de função importante a ser ressaltado neste quadro é o fígado. O mesmo é o principal órgão responsável pelo metabolismo de carboidratos, proteínas, lipídeos e xenobióticos no corpo humano, sendo ele também responsável por produzir bile, além de diversas proteínas e lipoproteínas. Uma vez lesado, diversas enzimas são dosadas no sangue periférico que determinam a extensão, grau de comprometimento e possíveis causas (PORTA G, et al., 2019). Desta forma, casos de hepatopatias graves podem agravar ainda mais o quadro clínico do paciente com COVID-19, e por este motivo, faz-se necessário a avaliação dos marcadores de função hepática. Dentre elas, destaca-se a enzima Gama-Glutamil Transferase (GGT) a sua elevação pode ser um indicativo de cirrose, por exemplo, que torna difícil o tratamento da covid-19 devido fazer com que o fígado perca sua função (BRANDÃO SCS, et al., 2020; PORTA G, et al., 2019).

De modo similar, tanto a AST, quanto a alanina aminotransferase (ALT) são enzimas citoplasmáticas hepáticas, responsáveis por fazer o metabolismo de aminoácidos e outras substâncias (OLIVEIRA JRB, et al., 2020). Por estarem presentes apenas no ambiente intracelular, níveis elevados das mesmas na corrente sanguínea corroboram com a morte dos hepatócitos, fato que acontece em diversas doenças hepáticas agudas e crônicas (WANG J, et al., 2020). Além disso, sabe-se que quanto maior a dosagem de AST e ALT, mairo o dano hepático, sendo em casos agudos, mais expressivos que casos crônicos devido a população geral de células afetadas. Assim, tratando-se especificamente da obesidade, sabe-se que a mesma é um fator de risco para desenvolvimento de esteatose hepática, bem como progressão do quadro para esteatohepatite e cirrose (LI J, et al., 2019).

Tal fato esta associado à alteração do metabolismo energético dos hepatócitos e acúmulo de ácidos graxos em seu interior, por exemplo (SARWAR R, et al., 2018). Neste mesmo raciocínio, já foi demonstrado casos de pacientes com COVID-19 que apresentaram elevação dos marcadores de dano hepático, concluindo-se que o SARS CoV-2 atinge também os hepatócitos e colangiócitos do fígado, além de causar resposta inflamatória sistêmica. Tendo como base o exposto, entende-se que a avaliação dos marcadores de dano e função hepática no paciente obeso com COVID-19 é relevante, devido o risco duplo aos hepatócitos causados pelo vírus e pela alteração metabólica (SANTOS RS, et al., 2020).

Fisiologicamente, o rim é um importante órgão do corpo humano responsável pela produção de urina, bem como eliminação de restos metabólitos de nutrientes e outros compostos no corpo humano (RAMOS $\mathrm{G} e$ MARINI DC, 2014). Visto que a disfunção renal é um dos eventos de complicação na COVID-19 e também na obesidade, é de suma importância avaliar a função renal deste paciente. Para esta finalidade, utilizam-se diversos marcadores específicos, que pela amostra de sangue e urina, indicam se as funções renais estão comprometidas totalmente ou somente diminuídas. Dentre estes marcadores têm-se os mais conhecidos como a ureia, creatinina, avaliação da taxa de filtração glomerular (TGF) de diversas substâncias, e dos elementos do sedimento da urina (EAS) (RAMOS G e MARINI DC, 2014; GANSEVOORT RT e HILBRANDS LB, 2020; HEYMSFIELD SB, et al., 2014).

Assim, a avaliação da função dos rins pode ser realizada através do soro presente no sangue onde são dosadas a ureia, creatinina, níveis de microglobulina e albumina, por exemplo. A formação da creatinina se dá através da creatina presente nos músculos, ocorrendo após a quebra e desidratação da creatina e sua excreção é realizada pelos rins, por isso quando há presença de uma quantidade maior que o normal de creatinina no soro entende-se como indicativo de que as funções estão de algum modo afetadas (MOURÃO $\mathrm{BCL}, 2019)$.

No sangue, o carbono e a amônia estão presentes na circulação, após a metabolização tem como produto final a ureia que é eliminada pela urina $A$ ureia em conjunto com a creatinina podem indicar diferenças nas patologias presentes, mas a creatinina continua sendo um marcador essencial por avaliar o ritmo da filtração. 
Por sua vez, a microglobulina é útil na identificação de problemas associados a menor reabsorção dos túbulos renais, enquanto que a albumina indica alterações associadas à glomerolunefrite ou insuficiência renal (MOURÃO BCL, 2019).

Outro importante marcador relevante é a taxa de filtração glomerular (TFG), uma vez que avalia a capacidade de filtração, reabsorção e secreção renal de diversas substâncias. Dentre elas, a cistatina C é uma das mais importantes, pois é estável, tem circulação constante por ser reabsorvida, não é dependente de sexo, massa muscular ou da alimentação que o paciente apresenta no seu dia a dia (MACEDO YM, et al., 2020). Neste mesmo sentindo, um importante aliado na avaliação do paciente com insuficiência renal é o sumário de urina (EAS).

Além da rapidez na avaliação e baixo custo, este teste avalia desde os aspectos físicos, químicos a achados anormais na urina, tais como sangue, fungos e bactérias. Por sua vez, havendo alterações, 0 paciente é encaminhado para exames mais específicos no intuito de descobrir o agente etiológico e tratamento específico (CABRAL JVR e PATRY KO, 2019). Nesta opção de exame pode-se encontrar um caso de proteinúria que também é um marcador renal importante, tem indicação de alteração de disfunção tubular (PALLET N, et al., 2018).

De acordo com a cartilha do Ministério da Saúde (2020), os pacientes com COVID-19 são avaliados e caso percebam a necessidade são prescritos os exames para avaliação renal e hepática. De modo geral, entende-se que tais padrões de avaliação são realizados rotineiramente em todo o país, entretanto, em vista da quantidade e gravidade dos casos de COVID-19 em todo o mundo, há a necessidade de uma maior atenção aos mesmos. Tendo o detalhamento dos principais exames e locais de avaliação, ressalta-se por fim que o paciente obeso geralmente possui outras patologias que levam a um pior prognóstico da COVID-19 no mesmo. Assim, é imprescindível que o laboratório tenha comunicação direta com a equipe médica responsável pelo acompanhamento do paciente para que se tenha uma melhor discussão sobre quais outros exames são complementares para a avaliação geral do paciente, bem como quais medidas podem ser adotadas visando a melhora do mesmo.

\section{CONSIDERAÇÕES FINAIS}

Compreende-se que os pacientes que apresentam obesidade têm risco de complicações elevado durante a infecção pelo SARS-CoV-2. Estes mesmos pacientes, em geral, podem ter comprometimento cardiovascular, renal e metabólicos sérios, sendo todos estes fatores amplificados de forma negativa durante a COVID-19. Atualmente, o acompanhamento dos mesmos por meio de exames laboratoriais é uma alternativa válida e necessária, uma vez são realizados na rotina laboratorial. Para tais avaliações, existem uma grande quantidade de exames que são realizados rotineiramente, entretanto, ressalta-se que há a necessidade de um profissional capacitado para a interpretação dos exames, evitando erros de interpretação e acompanhamento. Por fim, entende-se que o paciente obeso com COVID-19 deve ser acompanhado por uma equipe multiprofissional, que possa avaliar o paciente de acordo com habilidades específicas de cada área, visando sempre sua melhora. A busca continínua pela atualização e comunicação entre equipes de saúde é de extrema importância neste cenário.

\section{REFERÊNCIAS}

1. BRANDÃO SCS, et al. Obesidade e risco de Covid-19: grave. 1 Ed., 2020, 113 p.

2. BRAY MS, et al. Using genomic information to guide weight management: From universal to precision treatment: TransNIH Conference on Genes, Behaviors and Response to Weight Loss Interventions. Obesity (Silver Spring, Md.), 2016; 24(1): 14-22.

3. CABRAL JVR, PATRY KO. A importância dos exames laboratoriais nos procedimentos estéticos. Revista Brasileira de Estética, 2019; 7(18): 1-14.

4. CERCATO C, FONSECA FA. Cardiovascular risk and obesity. Diabetology \& metabolic syndrome, 2019; 11(1): 1-15.

5. COLARES VS, et al. COMDORA-SBN recommendations for patients with rare kidney diseases in relation to the Covid19 pandemic. Brazilian Journal of Nephrology, 2020; 42(2): 36-40.

6. FÖLDI M, et al. Visceral adiposity elevates the risk of critical condition in COVID-19: A systematic review and metaanalysis. Obesity, 2021; 29(3): 521-528. 
7. FREIRE EG, et al. Efeito da Disfunção Renal Crônica Sobre a Permeabilidade do Cólon para Água e Eletrólitos: Estudo Experimental em Ratos. Arquivos Brasileiros de Cirurgia Digestiva, 2019; 32(4): e1472.

8. GANSEVOORT RT, HILBRANDS LB. CKD is a key risk factor for COVID-19 mortality. Nature Reviews Nephrology; 2020; 16(12): 705-706.

9. GUERRA JDMB, et al. Análise de Biomarcadores em Lesões Incipientes e Crônicas. Trabalho de Conclusão de Curso (Medicina) - Instituto de Educação Superior do Vale do Parnaíba (IEVASPI), Piauí, Parnaíba, 2020; 14p.

10. HEYMSFIELD SB, et al. Weight loss composition is one-fourth fat-free mass: a critical review and critique of this widely cited rule. Obesity Reviews, 2014; 15(4): 310-321.

11. HEYMSFIELD SB; WADDEN TA. Mechanisms, pathophysiology, and management of obesity. New England Journal of Medicine , 2017; 376(3): 254-266.

12. HU B, et al. Characteristics of SARS-CoV-2 and COVID-19. Nature Reviews Microbiology, 2021; 19(3): $141-154$.

13. JONAS A. O aumento da obesidade em crianças e adolescentes e seus principais fatores determinantes. Faculdade de Ciências da Saúde-FAEF. Psicologia. PT-O Portal dos Psicólogos, 2018: 1-9.

14. KAUR J. A comprehensive review on metabolic syndrome. Cardiology research and practice, 2014.

15. KIRCH JR. Obesidade, causas e o tratamento á luz da psicanálise - Universidade Regional do Noroeste do estado do Rio Grande so Sul - UNIJUI. Rio Grande do Sul - ljuí, 2017.

16. LI J, et al. Prevalence, incidence, and outcome of non-alcoholic fatty liver disease in Asia, 1999-2019: a systematic review and meta-analysis. The lancet Gastroenterology \& hepatology, 2019; 4(5): 389-398.

17. MACEDO YM, et al. COVID-19 No Brasil: o que se espera para população subalternizada?. Revista EncantarEducação, Cultura e Sociedade, 2020; 2: 1-10.

18. MANNA P; JAIN SK. Obesity, Oxidative Stress, Adipose Tissue Dysfunction, and the Associated Health Risks: Causes and Therapeutic Strategies. Metab Syndr Relat Disord, 2015; 3(10): 423-444.

19. MANUAL DE EXAMES LABORATÓRIAIS: rede SUS-BH. Belo Horizonte: Gerência de Secretaria Municipal de Saúde - Comunicação Visual. (Brasil), 2016.

20. MINISTÉRIO DA SAÚDE. Secretaria de atenção Especializada à Saúde. Departamento de Atenção Hospitalar, Domiciliar e de Urgência. Protocolo de manejo clínico da Covid-19 na atenção Especializada - 1ª edição, 48 p - Revista Brasília - DF: Brasil, 2020. Disponível em: https://bvsms.saude.gov.br/bvs/publicacoes/manejo_clinico_covid19_atencao_especializada.pdf. Acesso em: 10/04/2021.

21. MOURÃO BCL, et al. Alterações renais relacionadas com desequilíbrios dos exames bioquímicos - Revista Saúde em Foco, Ed. 11; 2019.

22. NISHIGA M, et al. COVID-19 and cardiovascular disease: from basic mechanisms to clinical perspectives. Nature Reviews Cardiology, 2020; 17(9): 543-558.

23. OLIVEIRA JUNIOR RB, LOURENÇO PM. Alterações laboratoriais e a COVID-19. Revista Brasileira de Análises Clínicas, 2020; 52(2):198-200.

24. PALLET N, et al. Proteinuria typing: how, why and for whom?. In: Annales de biologie clinique. 2019: $13-25$.

25. PORTA G, et al. Autoimmune hepatitis in 828 Brazilian children and adolescents: clinical and laboratory findings, histological profile, treatments, and outcomes. Jornal de pediatria, 2019; 95(4): 419-427.

26. RAMOS G, MARINI DC. Exames bioquímicos relacionados a alterações renais. FOCO: caderno de estudos e pesquisas, $2014 ; 6$.

27. REIS MAOM, et al. Complicações cardiovasculares em pacientes com Diabetes Mellitus Tipo 2. Revista Eletrônica Acervo Saúde, 2021; 13(3), e6426.

28. SANTI MC. Efeito da suplementação de creatina sobre marcadores de lesão muscular e desempenho físico em atletas de voleibol. Universidade de São Paulo. Ribeirão Preto, 2018.

29. SANTOS RS, et al. Achados ultrassonográficos abdominais em pacientes com COVID-19. Sociedade Brasileira de Ultrassonografia, 2020; 4(1);1-5.

30. SARWAR R, et al. Obesity and nonalcoholic fatty liver disease: current perspectives. Diabetes, metabolic syndrome and obesity: targets and therapy, 2018; 11:533.

31. SILVA CC, et al. Covid-19: Aspectos da origem, fisiopatologia, imunologia e tratamento - uma revisão narrativa. Revista Eletrônica Acervo Saúde, 2021; 13(3): e6542.

32. SOCIEDADE BRASILEIRA DE CARDIOLOGIA (SBC) - Grupo de Especialistas em Biomarcadores. Biomarcadores em Cardiologia - Parte 1 - Na Insuficiência Cardíaca e nas Cardiomiopatias Específicas. Arq Bras Cardiologia. 2014; 103(6):451-459. Disponível em: https://www.scielo.br/j/abc/a/XcKSqBhqDcJjK7KMyxJFmzc/?lang=pt. Acesso em: $16 / 03 / 2021$.

33. SOCIEDADE BRASILEIRA DE CARDIOLOGIA (SBC) - Grupo de Especialistas em Biomarcadores. Biomarcadores em Cardiologia - Parte 2: Na Doença Coronária, Doença Valvar e Situações Especiais. Arq Bras Cardiologia. 2015; 104(5):337-346. Disponível em: https://www.scielo.br/j/abc/a/KZPQQv7DqsPGZJFDYjSpXCn/?lang=pt. Acesso em: $16 / 03 / 2021$

34. SOCIEDADE BRASILEIRA DE DIABETES (SBD). Diretrizes da Sociedade Brasileira de Diabetes: 2019-2020. Brasil, [S. I.]: Clannad - Editora Científica, 2019, 491p. Disponível em: http://www.saude.ba.gov.br/wpcontent/uploads/2020/02/Diretrizes-Sociedade-Brasileira-de-Diabetes-2019-2020.pdf. Acesso em: 20/03/2021.

35. SOUSA ELH. Marcadores bioquímicos e genéticos na predição de fatores de risco para doenças cardiovasculares em jovens universitários brasileiros e africanos. 2020. $90 \mathrm{f}$. Tese (Doutorado em Biotecnologia) - Universidade Federal do Ceará, Fortaleza, 2020.

36. TAY MZ, et al. The trinity of COVID-19: immunity, inflammation and intervention. Nature Reviews Immunology, 2020; 20(6): 363-374.

37. WANG J, et al. Association between alanine aminotransferase/aspartate aminotransferase ratio (AST/ALT Ratio) and coronary artery injury in children with Kawasaki disease. Cardiology research and practice, 2020.

38. XU Z, et al. Pathological findings of COVID-19 associated with acute respiratory distress syndrome. The Lancet respiratory medicine, 2020; 8(4): 420-422.

39. ZHU N, et al. A novel coronavirus from patients with pneumonia in China, 2019. New England journal of medicine, 2020. 